\title{
Modifiable cardiovascular disease risk factors as predictors of dementia death: pooling of ten general population-based cohort studies
}

\author{
G David Batty ${ }^{1,2,3^{*}}$, Tom C Russ ${ }^{2,3,4,5}$, John M Starr ${ }^{2,3}$, Emmanuel Stamatakis ${ }^{1}$ and Mika Kivimäki ${ }^{1}$
}

\begin{abstract}
Background: With drug treatment for dementia being of limited effectiveness, the role of primary prevention, in particular the predictive value of modifiable cardiovascular disease risk factors, may warrant exploration. The evidence base is, however, characterised by discordant findings and is modest in size. Accordingly, we examined the association of modifiable cardiovascular disease risk factors with dementia death.

Design and methods: We pooled raw data from 10 UK general population-based prospective cohort studies within the context of an individual participant meta-analysis.

Results: A total of 103,764 men and women were followed up for a mean of 8 years giving rise to 443 dementia-related deaths and 2612 cardiovascular disease deaths. Cardiovascular disease mortality was, as anticipated, associated with the full range of risk factors under study, including raised blood pressure, smoking, diabetes, physical inactivity. By contrast, dementia death was related to very few of the cardiovascular disease risk factors: of those classified as modifiable, only smoking was associated with a raised risk and higher levels of non-HDL with a lower risk.
\end{abstract}

Conclusions: In the present individual participant meta-analysis, there was limited evidence that cardiovascular disease risk factors were related to dementia death.

\section{Background}

The present and future social, economic, and disease burden exacted by dementia is considerable and well documented. That drug treatment, even when implemented in the early stages of the illness, is only symptomatic and of limited effectiveness raises the need to identify primary risk factors for dementia. If modifiable environmental indices are shown to predict dementia, this could have implications for control of the disease.

In particular, it has been posited that cardiovascular disease (CVD) risk factors, many of which are modifiable, are linked to dementia development. The notion that CVD and dementia or cognitive impairment may have a shared aetiology was first advanced over four decades ago [1]. However, in a recent consensus statement,

\footnotetext{
* Correspondence: david.batty@ucl.ac.uk

'Department of Epidemiology and Public Health, University College, 1-19

Torrington Place, London, UK

${ }^{2}$ Centre for Cognitive Ageing \& Cognitive Epidemiology, University of

Edinburgh, Edinburgh, UK

Full list of author information is available at the end of the article
}

an expert panel concluded that for Alzheimer's Disease, which represents up to $70 \%$ of all dementia cases [2], there is insufficient evidence with which to form firm conclusions about the influence of any modifiable environmental characteristic, including CVD risk factors [3].

Accordingly, we examined the association of a range of established CVD risk factors with dementia death. To do so, as we have done in different contexts [4-7], we present a meta-analysis of individual-participant data from ten UK population-based cohort studies. Taking this approach involves the pooling of raw data. In contrast to the more traditional literature-based meta-analysis in which investigators may have to exclude publications that do not present results in a standard manner, the possibility of publication bias is reduced in an individual participant meta-analysis. Additionally, a literature based meta-analysis cannot provide a consistent approach to statistical control for plausible covariates. While the individual participant meta-analysis approach has been taken for physiological risk factors in relation to somatic disease outcomes [8,9], it is much less common in the 
field of psychiatry. To our knowledge, this is the largest study to date to explore the link between individual CVD risk factors and dementia death and the first individual participant meta-analysis. For the purposes of comparison, we also report on the relation of the same collection of risk factors with CVD mortality.

\section{Methods}

The Health Surveys for England (conducted 1998, 1999, 2003-6, and 2008) [10] and the Scottish Health Surveys (1995, 1998, and 2003) [11] are UK-representative, repeat, cross-sectional, independent studies with on-going mortality surveillance of their study members. Participants gave informed consent; ethical approval was obtained from the London Research Ethics Council. Participants completed a questionnaire and took part in clinical examination. During both, CVD risk factors were assessed using standard operating protocols as have been described in detail [5,7].

Participants were linked to national mortality registers. Causes of death (up to ten) recorded on certificates were coded using the International Classification of Diseases, Ninth (ICD-9) [12] and Tenth (ICD-10) [13] revisions. Any mention of dementia death was identified using codes 290.0 to 290.4, 294.9, 331.0 to 331.2, and 331.9 for ICD-9 and F00, F01, F03, F09, G30 and G31 for ICD-10. We have previously shown that in a cohort of Scottish individuals with psychiatrist-confirmed dementia, almost three quarters of those who subsequently died had dementia recorded on their death certificate [14]. This suggests that the use of dementia deaths data has some validity.

\section{Statistical analyses}

Having ascertained that the proportional hazards assumption had not been violated, we then used Cox proportional hazards models [15] to compute study-specific hazard ratios with accompanying 95\% confidence intervals for the association between each CVD risk factor and dementia death. Preliminary results suggested that there was no consistent evidence for differential risk factordementia effects by gender; data for men and women were therefore pooled with hazard ratios sex-adjusted.

The study-specific effect estimates and their standard errors were pooled using a random effects meta-analysis. In contrast to a fixed-effects meta-analysis, a randomeffects approach allows for variation in observed risk factor-disease estimates across studies which occur because of real differences in the association in each study and/or sampling variability. We chose $a$ priori a random-effects technique for two reasons. First, many of the CVD risk factors featured in the present report (raised blood pressure, obesity, physical activity, smoking and so on) have been the subject of primary care-based modification during the period of follow-up of the included studies. Depending on the era of the study in question, this may have had an impact on the risk factor-disease associations under scrutiny. Second, there have now been a series of papers from this collaboration in which the outcomes of interest have been the same as the present analyses-CVD $[5,7]$ and dementia [4-6]-although the predictor variables have differed. That these existing results suggest evidence of heterogeneity across studies, provided us with a second reason to utilise the random-effects approach.

Heterogeneity in the effect estimates between studies was examined by computing an $\mathrm{I}^{2}$ statistic, which indicates the proportion of the total variation in the estimates that is due to between-studies variation. Calendar time (months) was the time scale. We censored study members at death or the end of follow-up (15th February 2011), whichever came first.

\section{Results}

From an initial sample of 122,060 participants, 18,296 did not consent to mortality record linkage or, if they did, had missing survival or mortality data, giving a maximum analytic sample of 103,764 (55\% female, mean age 47.3 years, $\mathrm{SD}=18.1$, range $=16-102)$. Of 7933 deaths occurring during a mean (SD) follow up of 7.9 (3.8) years, 443 were dementia-related and 2612 were cardiovascular disease-related. With dementia as the outcome of interest, the $\mathrm{I}^{2}$ statistic varied between $0 \%$ and $76.4 \%$ depending on the CVD risk factor in question. This provided post hoc justification of our random-effects approach to the metaanalysis.

Table 1 depicts the association of fourteen CVD risk factors with dementia death and CVD death risk. Importantly, known risk factor-CVD relationships were all replicated in the present analyses. Thus, conventionally unfavourable levels of blood pressure and total cholesterol, high body mass index, smoking, diabetes and low physical activity were associated with higher CVD death rates, although effect of systolic hypertension did not reach statistical significance.

By contrast, dementia was associated with very few of the CVD risk factors. Being male, older, and smoking were shown to confer an increased risk of dementia death, while higher non-HDL cholesterol and obesity were both associated with protection. The result for obesity was surprising. Speculating that this could be ascribed to reverse causality-dementia influencing the risk factor rather than the converse-led us to drop dementia deaths occurring in the first five years of follow-up ('left' censoring) and repeat our analyses (a standard analytical procedure in epidemiology). This attenuated the obesity-dementia association to the null (HR; 95\% CI: 0.88 ; $0.62-1.26$ based on 214 dementia deaths in 70391 men and women). 
Table 1 Age- and gender-adjusted hazard ratios (95\% confidence intervals) for the relation of individual cardiovascular disease risk factors with dementia death and cardiovascular disease death: individual participant meta-analysis of ten general population-based cohort studies

\begin{tabular}{|c|c|c|c|c|c|c|c|c|c|}
\hline \multirow[t]{2}{*}{ Risk factor } & \multirow[t]{2}{*}{ Comparison } & \multicolumn{4}{|c|}{ Cardiovascular disease } & \multicolumn{4}{|c|}{ Dementia } \\
\hline & & Deaths & $\mathrm{N}^{\mathrm{k}}$ & $\mathrm{HR}(95 \% \mathrm{Cl})$ & P-value & Deaths & $N^{k}$ & HR $(95 \% \mathrm{Cl})$ & P-value \\
\hline Age & Per 5 yr increase & 2612 & 103764 & $1.78(1.74,1.82)$ & $<0.001$ & 443 & 103764 & $2.42(2.29,2.56)$ & $<0.001$ \\
\hline Gender & Female vs. male & 2612 & 103764 & $0.60(0.53,0.68)$ & $<0.001$ & 443 & 103764 & $0.78(0.65,0.95)$ & 0.01 \\
\hline Marital status & See footnote ${ }^{a}$ & 990 & 61171 & $1.34(1.00,1.80)$ & 0.049 & 156 & 61171 & $0.94(0.58,1.53)$ & 0.81 \\
\hline Occupational social class & Non-manual vs. manual & 2486 & 97455 & $1.36(1.18,1.57)$ & $<0.001$ & 424 & 97455 & $1.11(0.86,1.43)$ & 0.42 \\
\hline Educational attainment & Left school $\leq 15$ yr vs. older & 2610 & 103680 & $1.38(1.25,1.52)$ & $<0.001$ & 443 & 103680 & $1.18(0.95,1.45)$ & 0.13 \\
\hline Hypertension & See footnote ${ }^{b}$ & 1600 & 63989 & $2.10(1.83,2.41)$ & $<0.001$ & 283 & 61751 & $0.96(0.71,1.30)$ & 0.79 \\
\hline Systolic blood pressure & See footnote ${ }^{c}$ & 1600 & 63989 & $1.08(0.90,1.30)$ & 0.39 & 283 & 61751 & $0.87(0.68,1.11)$ & 0.27 \\
\hline Diastolic blood pressure & See footnote ${ }^{d}$ & 1600 & 63989 & $1.50(1.31,1.71)$ & $<0.001$ & 283 & 61751 & $1.02(0.69,1.50)$ & 0.92 \\
\hline Uncontrolled hypertension & See footnote $e^{e}$ & 803 & 46410 & $2.55(2.08,3.12)$ & $<0.001$ & 128 & 44626 & $0.88(0.60,1.29)$ & 0.52 \\
\hline Smoking status & Current smoker vs. other & 2606 & 103282 & $1.86(1.62,2.15)$ & $<0.001$ & 443 & 103282 & $1.75(1.33,2.29)$ & $<0.001$ \\
\hline Current drinker & Current vs. never/former ${ }^{f}$ & 2610 & 103072 & $0.77(0.64,0.94)$ & 0.01 & 443 & 103072 & $0.85(0.67,1.07)$ & 0.17 \\
\hline High total cholesterol & See footnote ${ }^{g}$ & 1424 & 55355 & $1.30(1.04,1.62)$ & 0.02 & 233 & 53375 & $0.88(0.67,1.15)$ & 0.34 \\
\hline Non-HDL cholesterol ${ }^{\text {h }}$ & Per SD (1.2 mmol/L) rise & 1135 & 49562 & $1.06(1.00,1.19)$ & 0.04 & 200 & 42271 & $0.82(0.70,0.96)$ & 0.01 \\
\hline Serum C-reactive protein & Per SD (6.7 mmol/L) rise & 1022 & 39352 & $1.14(1.07,1.21)$ & $<0.001$ & 191 & 37439 & $1.03(0.93,1.15)$ & 0.56 \\
\hline Body mass index & $\geq 30 \mathrm{~kg} / \mathrm{m}^{2}$ vs. $<30$ & 1952 & 90978 & $1.24(1.12,1.37)$ & $<0.001$ & 322 & 90978 & $0.71(0.53,0.97)$ & 0.03 \\
\hline Diabetes status & Yes vs. no & 2605 & 100639 & $1.97(1.65,2.35)$ & $<0.001$ & 441 & 100639 & $1.03(0.73,1.46)$ & 0.85 \\
\hline Physical inactivity & $<5$ sessions/week vs. $\geq 5^{j}$ & 2404 & 95426 & $1.63(1.40,1.89)$ & $<0.001$ & 401 & 95426 & $1.17(0.72,1.91)$ & 0.52 \\
\hline Longstanding illness & Any vs. none & 2612 & 103742 & $1.91(1.74,2.09)$ & $<0.001$ & 443 & 103742 & $1.10(0.90,1.36)$ & 0.35 \\
\hline
\end{tabular}

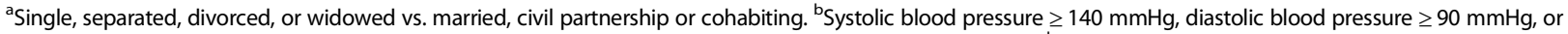
taking antihypertensive treatment (NICE guidance) vs. other. ${ }^{\mathrm{C} S y s t o l i c}$ blood pressure $\geq 140 \mathrm{mmHg}$ vs other. ${ }^{\mathrm{d}}$ Diastolic blood pressure $\geq 90 \mathrm{mmHg}$ vs other. ${ }^{\mathrm{e}} \mathrm{Despite}$ antihypertensive medication, either systolic blood pressure $\geq 140 \mathrm{mmHg}$, diastolic blood pressure $\geq 90 \mathrm{mmHg}$, or both vs. normotensive individuals. ${ }^{\mathrm{f}} \mathrm{Current}$ drinkers (any amount) compared to never- or ex-drinkers. ${ }^{9}$ Serum total cholesterol $\geq 6.2 \mathrm{mmol} / \mathrm{L}$ or on lipid-lowering treatment versus other. ${ }^{\mathrm{h}} \mathrm{Non}-\mathrm{HDL}$ cholesterol (calculated by subtraction of HDL-C from total cholesterol, yielding a measure that encompasses low-, intermediate-, and very-low-density lipoprotein cholesterol). Hazard ratios are per standard deviation increase (disadvantage). 'Diabetes denoted by doctor-diagnosed diabetes, longstanding illness (diabetes), $\mathrm{HbA}_{1 c} \geq 6.0 \%$, and/or diabetes medication. ${ }^{\mathrm{j}}$ Fewer than five average weekly sessions of moderate to vigorous physical activity including domestic (walk/domestic 30 mins+, sports/exercise 15 mins+) compared to five or more (UK government recommendations). ${ }^{\mathrm{k}}$ Some cohort studies were excluded from specific risk factor analyses owing to the absence of any dementia deaths.

Finally, for the four CVD risk factors found to be associated with dementia death in the above age- and genderadjusted analyses we carried out mutual adjustment. Based on 322 dementia deaths in the 60625 men and women with complete data, and using the same comparators, our results were essentially unchanged: age $(2.53 ; 2.35,2.73)$, gender $(0.80 ; 0.64,1.00)$, smoking $(1.60 ; 1.15,2.22)$ and body mass index $(0.72 ; 0.53,0.97)$.

\section{Discussion}

In the present study-to our knowledge, the first metaanalysis in the field-there was limited evidence that CVD risk factors were related to an increased occurrence of dementia death. The few positive results included higher age, male sex, smoking and, surprisingly being non-obese, the last finding disappearing when taking into account reverse causality. That established risk factor-CVD associations were confirmed, gives us some confidence in the results for dementia.

While our dataset has its strengths-its size and the comprehensive set of CVD risk factors variables assessed-it is of course not without its shortcomings. While we had data on a series of health behaviours (smoking, alcohol intake, and exercise) we did not have information on dietary characteristics which may be linked to dementia risk. Additionally, comprising a series of subtypes (Alzheimer's disease, vascular dementia, AIDS dementia, and so on), dementia is not a single nosological entity. As it is likely that these subtypes do not have a unifying aetiology, using a composite dementia endpoint may mask some subtype-specific associations with CVD risk factors. It is also the case, however, that, with the majority of dementias being Alzheimer's, our findings are most applicable to this dementia presentation. Finally, our outcome of interest was dementia death, not incidence; as such, our exposures are more distant from dementia event. This notwithstanding, as described, in a separate study [14], we found that, in a cohort of patients diagnosed with probable Alzheimer's disease, of the 502 who had died after 11 years of follow-up, $72 \%$ had their dementia recorded as either a primary or contributing cause on their death certificate. This 
suggests that using dementia death may approximate dementia incidence.

Taking these results together, there was limited evidence that these CVD risk factors were related to the occurrence of dementia death in the current study.

\section{Competing interests}

The authors declare that they have no competing interests.

\section{Author contributions}

GDB conceived the idea for the present manuscript; ES built the dataset; and TCR carried out the data analyses. GDB, TCR and MK interpreted the results, and GDB wrote the first draft of the manuscript on which all authors provided comments. All authors read and approved the final manuscript for submission.

\section{Funding}

GDB, JMS and TCR are members of both the Alzheimer Scotland Dementia Research Centre (funded by Alzheimer Scotland), and the University of Edinburgh Centre for Cognitive Ageing and Cognitive Epidemiology, part of the cross council Lifelong Health and Wellbeing Initiative (funded by the BBSRC, EPSRC, ESRC, and MRC). MK is supported by the Medical Research Council (K013351), the Economic and Social Research Council, UK, the Finnish Work Environment Fund, and the National Institute on Aging, $\mathrm{NIH}$, USA (R01 AG034454, R01AG013196, R01HL036310).

\section{Author details}

'Department of Epidemiology and Public Health, University College, 1-19 Torrington Place, London, UK. ${ }^{2}$ Centre for Cognitive Ageing \& Cognitive Epidemiology, University of Edinburgh, Edinburgh, UK. ${ }^{3}$ Alzheimer Scotland Dementia Research Centre, University of Edinburgh, Edinburgh, UK. ${ }^{4}$ Scottish Dementia Clinical Research Network, NHS Scotland, Edinburgh, UK. 'Division of Psychiatry, University of Edinburgh, Edinburgh, UK.

Received: 3 March 2014 Accepted: 6 May 2014

Published: 23 May 2014

\section{References}

1. Wilkie F, Eisdorfer C: Intelligence and blood pressure in the aged. Science 1971, 172(3986):959-962.

2. Lobo A, Launer L, Fratiglioni L, Andersen K, Di CA, Breteler MM, Copeland JR, Dartigues JF, Jagger C, Martinez-Lage J, Soininen H, Hofman A: Prevalence of dementia and major subtypes in Europe: a collaborative study of population-based cohorts. Neurologic Diseases in the Elderly Research Group. Neurology 2000, 54(11 Suppl 5):S4-S9.

3. Daviglus ML, Bell CC, Berrettini W, Bowen PE, Connolly ES Jr, Cox NJ, Dunbar-Jacob JM, Granieri EC, Hunt G, McGarry K, Patel D, Potosky AL, Sanders-Bush E, Silberberg D, Trevisan M: National Institutes of Health State-of-the-Science Conference statement: preventing Alzheimer disease and cognitive decline. Ann Intern Med 2010, 153(3):176-181.

4. Russ TC, Stamatakis E, Hamer M, Starr JM, Kivimaki M, Batty GD: Socioeconomic status as a risk factor for dementia death: individual participant meta-analysis of 86508 men and women from the UK. Br J Psychiatry 2013, 203:10-17.

5. Russ TC, Hamer M, Stamatakis E, Starr JM, Batty GD, Kivimaki M: Does the Framingham cardiovascular disease risk score also have predictive utility for dementia death? An individual participant meta-analysis of 11,887 men and women. Atherosclerosis 2013, 228(1):256-258,

6. Russ TC, Hamer M, Stamatakis E, Starr JM, Batty GD: Psychological distress as a risk factor for dementia death. Arch Intern Med 2011, 171(20):1858-9.

7. Russ TC, Stamatakis E, Hamer M, Starr JM, Kivimaki M, Batty GD: Association between psychological distress and mortality: individual participant pooled analysis of 10 prospective cohort studies. BMJ 2012, 345:e4933.

8. Lewington S, Clarke R, Qizilbash N, Peto R, Collins R: Age-specific relevance of usual blood pressure to vascular mortality: a meta-analysis of individual data for one million adults in 61 prospective studies. Lancet 2002, 360(9349):1903-1913.

9. Emerging Risk Factors Collaboration, Wormser D, Kaptoge S, Di Angelantonio E, Wood AM, Pennells L, Thompson A, Sarwar N, Kizer JR, Lawlor DA, Nordestgaard BG, Ridker P, Salomaa V, Stevens J, Woodward M,
Sattar N, Collins R, Thompson SG, Whitlock G, Danesh J: Separate and combined associations of body-mass index and abdominal adiposity with cardiovascular disease: collaborative analysis of 58 prospective studie. Lancet 2011, 377(9771):1085-95.

10. Mindell J, Biddulph JP, Hirani V, Stamatakis E, Craig R, Nunn S, Shelton N: Cohort profile: the health survey for England. Int J Epidemiol 2012, 41(6):1585-1593.

11. Gray L, Batty GD, Craig P, Stewart C, Whyte B, Finlayson A, Leyland AH: Cohort profile: the Scottish health surveys cohort: linkage of study participants to routinely collected records for mortality, hospital discharge, cancer and offspring birth characteristics in three nationwide studies. Int J Epidemiol 2010, 39(2):345-350.

12. Anon: Manual of the International Statistical Classification of Diseases, Injuries, and Causes of Death (ninth revision). Geneva: WHO; 1977.

13. Anon: International Statistical Classification of Diseases and Related Health Problems (tenth revision). Geneva: WHO; 1992.

14. Russ TC, Batty GD, Starr JM: Cognitive and behavioural predictors of survival in Alzheimer disease: results from a sample of treated patients in a tertiary-referral memory clinic. Int I Geriatr Psychiatry 2012, 27(8):844-853.

15. Cox DR: Regression models and life-tables. J R Stat Soc Ser B 1972, $34: 187-220$

doi:10.1186/1477-5751-13-8

Cite this article as: Batty et al: Modifiable cardiovascular disease risk factors as predictors of dementia death: pooling of ten general population-based cohort studies. Journal of Negative Results in BioMedicine 2014 13:8.

\section{Submit your next manuscript to BioMed Central and take full advantage of:}

- Convenient online submission

- Thorough peer review

- No space constraints or color figure charges

- Immediate publication on acceptance

- Inclusion in PubMed, CAS, Scopus and Google Scholar

- Research which is freely available for redistribution 